\title{
Tangence
}

\section{Écriture et gains financiers : démarche contradictoire des acteurs du sous-champ de la littérature jeunesse au Québec ?}

\section{Jean-Denis Côté}

Numéro 67, automne 2001

L'écriture pour la jeunesse : de la production à la réception

URI : https://id.erudit.org/iderudit/009614ar

DOI : https://doi.org/10.7202/009614ar

Aller au sommaire du numéro

Éditeur(s)

Presses de l'Université du Québec

ISSN

0226-9554 (imprimé)

1710-0305 (numérique)

Découvrir la revue

Citer cet article

Côté, J.-D. (2001). Écriture et gains financiers : démarche contradictoire des acteurs du sous-champ de la littérature jeunesse au Québec ? Tangence, (67), 34-53. https://doi.org/10.7202/009614ar
Résumé de l'article

Résumé

Existe-t-il une contradiction entre réaliser une oeuvre d'art, en l'occurrence une oeuvre littéraire, et rechercher un gain d'ordre financier? Cette question est au coeur de la théorie du champ de l'art que propose le sociologue Pierre Bourdieu. Nous l'avons reprise à l'occasion d'une enquête sociologique menée auprès d'une trentaine d'acteurs de ce sous-champ : auteurs, auteurs/ éditeurs, professeurs d'université, critiques littéraires, animateurs de lecture. Les réponses des intervenants peuvent varier selon leur position d'acteur dans le sous-champ de la littérature jeunesse et selon le public auquel ils s'adressent. 


\section{Écriture et gains financiers : démarche contradictoire des acteurs du sous-champ de la littérature jeunesse au Québec?}

Jean-Denis Côté, Université Laval

Existe-t-il une contradiction entre réaliser une cuuve d'art, en l'occurrence une ceuvre littéraire, et rechercher un gain d'ordre financier? Cette question est au coeur de la théorie du champ de l'art et, plus précisément, de celui de la littérature, que propose Pierre Bourdieu. Pour le sociologue français, la vitalité ou la dynamique d'un champ est liée de manière intrinsèque à la lutte que se livrent les acteurs. Bourdieu précise que ces champs "sont le lieu de la coexistence antagoniste de deux modes de production et de circulation obéissant à des logiques inverses". À un pôle, on retrouve un mode de production commercial, propre aux industries culturelles, qui repose sur la même logique économique que les autres produits. Dans ce mode de production, on accorde la priorité à la diffusion, au succès rapide et éphémère, qui se vérifie par le tirage. Les entreprises qui optent pour ce mode de production sont attentives à la demande de la clientèle mais, en raison de leur appartenance à un champ qui leur procure un minimum de capital symbolique, s'abstiennent "de déclarer complètement leurs fins intéressées ${ }^{1}$. À l'autre pôle, des entreprises choisissent un mode de production qui se veut une négation de la logique commerciale en adoptant celle de la concurrence basée sur la reconnaissance "proprement culturelle accordée par le groupe des pairs, qui sont à la fois des clients privilégiés et des concurrents ${ }^{2}$. Ce mode de production favorise l'obtention de capital symbolique "comme capital "économique" dénié, reconnu, donc légitime" qui, "sous certaines conditions et à long terme ", peut se traduire en profits commerciaux ${ }^{3}$.

1. Pierre Bourdieu, Les règles de lart. Genèse et structure du champ littéraire, Paris, Seuil, 1992, p. 203.

2. Pierre Bourdieu, "Le marché des biens symboliques", L'année sociologique, Paris, vol. 22, 1971, p. 55.

3. Pierre Bourdieu, Les règles de l'art, ouvr. cité, p. 202. 
Ces deux pôles correspondent respectivement au champ de grande production symbolique et au champ de production restreinte. Pour saisir concrètement les résultats de tels modes de fonctionnement dans le champ de la littérature générale, ou voir comment ils s'inscrivent dans la réalité sociale, on pourrait donner l'exemple de deux éditeurs exprimant, selon nous, chacune des deux tendances: les éditions Harlequin, dont les romans sont bâtis d'après un canevas déterminé et uniforme ${ }^{4}$, lequel favorise non seulement l'accès à un public le plus large possible, mais répond à une demande préexistante ${ }^{5}$; les éditions des Herbes rouges, qui se consacrent essentiellement à la poésie et qui destinent leur production à un public forcément restreint ${ }^{6}$.

Est-ce à dire que les acteurs du champ de production restreinte s'opposent à une démocratisation de la culture en vertu de la "distinction" que leur procure la réalisation ou l'appropriation "matérielle ou symbolique d'une œuvre d'art " ${ }^{7}$ ? De fait, "les intellectuels et les artistes entretiennent avec tout ce qui touche à la "démocratisation de la culture" une relation d'extrême ambivalence $^{8}$ " qui se manifeste par la tenue d'un double discours concernant les institutions de diffusion culturelle et le public:

Interrogés sur les améliorations à apporter à la présentation des ceuvres dans les musées et, en particulier, sur l'opportunité des innovations pédagogiques visant à abaisser le "niveau d'offre" des ceuvres en fournissant des éclaircissements techniques, historiques ou esthétiques, les membres de la classe dominante et tout spécialement les professeurs et les spécialistes d'art essaient d'échapper à la contradiction en opérant une dissociation entre ce qui est soubaitable pour les autres et ce qui est soubaitable pour eux-mêmes. C'est parce que le musée est

4. Voir, à ce sujet, Julia Bettinotti, La corrida de l'amour. Le roman Harlequin, Montréal, Université du Québec à Montréal, coll. "Les cahiers du département d'études littéraires ", $n^{\circ} 6,1986,161 \mathrm{p}$.

5. Pierre Bourdieu, Les règles de l'art, ouvr. cité, p. 203: «Une entreprise est d'autant plus proche du pôle "commercial" que les produits qu'elle offre sur le marché répondent plus directement ou plus complètement à une demande préexistante, et dans des formes préétablies".

6. Lire, à ce sujet, Roger Chamberland, Circa les Herbes rouges. Modernité, postmodernité et avant-garde, thèse de doctorat, Université Laval, Québec, 1987, $336 \mathrm{f}$.

7. Pierre Bourdieu, La distinction. Critique sociale du jugement, Paris, Minuit, 1979 , p. 254.

8. Pierre Bourdieu, La distinction, ouvr. cité, p. 254. 
comme il est qu'il est leur privilège exclusif; il est donc comme il faut pour des gens comme eux, c'est-à-dire pour cles gens faits pour lui. Changer le musée, pour le rendre plus accessible, c'est donc leur enlever quelque chose, une part de leur mérite, de leur rareté. Mais ils ne peuvent manquer d'être sensibles au fait qu'on les interroge en priorité, eux, les familiers, sur la politique à suivre, parce que c'est leur reconnaître le privilège d'accorder aux autres une part de leur privilège. En acceptant les améliorations pédagogiques, c'est leur musée, celui qu'ils étaient seuls capables d'avoir, c'est-à-dire leur musée austère, ascétique et noble, qu'ils acceptent de livrer aux autres' 9 .

Mais au regard de ces distinctions qu'introduit la théorie des champs de Bourdieu, où se situent les œuvres de la littérature jeunesse québécoise? Comment fonctionne la dynamique de ce souschamp ou, plus précisément, comment s'y positionnent les acteurs? Certes, il est difficile de répondre de manière pleinement satisfaisante à de telles interrogations à l'intérieur du cadre restreint d'un article ${ }^{16}$. Toutefois, la question que je posais en introduction: existe-t-il une contradiction entre réaliser une ouvre d'art, en l'occurrence une ceuvre littéraire, et rechercher un gain d'ordre finan-

9. Pierre Bourdieu, La distinction, onvr cité, p. 254.

10. Cé n'est pas non plus le liéu de procéder à une recension exhaustive de tout ce qui s'ést pulblié sur cette question. Mentionnons cependant trois ouvrages d'importance, de même qu'un numéro de revue fort significatif. Concernant l'historire du sous-champ de la littérature jeunesse, voir Édith Madore, La littêrature pour la jeunesse au Québec, Montréal, Boréal, coll. "Boréal express", 1994, 127 p. Bien qu'il n'aborcle pas la littérature jeunesse à partir du concep de champ, l'imposiant ouvrage de Françoisc Lepage, le meilleur à ce jour portant sur la littérature jeunesse au pays, me semble incontournable: Françoise Lepage, Histoire de la littérature pour la jeunesse. Quêbec et francopbonies du Canada, suivic du Dictionnaire des atteurs et des illustrateurs, Orléans (Ontario), Éditions David, 2000, 826 p. Sur l'histoire plus spécifique cles éditions Médiaspaul et de leur secteur jeunesse, lire Josée Marcoux, Iittejrature jeunessé au Québec. Médiaspaul (Éditions Paulines 1947-1955), Montréal, Médiaspaul, 2000,239 p. Pour un portrait récent de la recherche universitaire sur le sujet, lire Voix et images, Montréal, $\mathrm{n}^{\prime} 74$ (Le champ littéraire de la jeunesse au carrefour de la recherche universitaire), hiver 2000. Coordonné par Jacques La Mothe, le dossier comprend cinq contributions: "Le concept dadolescence : évolution et représentation dans la littérature québécoise pour la jeunesse", de Françoise Lepage; "Langue et idéologie dans les textes de Gabriclle Roy publiês pour les enfants", de Claude Romney; "Chronotopes du roman québécois pour adolescents", de Claire Le Brun; "Discours cle l'adolescente dans le récit de jeunesse contemporain : l'exemple de Marie-Francine Hébert ", de Iucie Guillemette; et "Le jeu de l'intertexte dans Le trésor de Brion ", de Jacques La Mothe. 
cier?, me semble bien cerner ou, du moins, résumer, les caractéristiques propres au mode de fonctionnement de chacun des pôles, lesquels oscillent entre la création d'une œuvre, d'une part, la production d'une œuvre et la recherche (ou non) d'un profit, d'autre part. Cette question recèle également un intérêt particulier, dans la mesure où l'écrivain pour la jeunesse, contrairement à l'écrivain pour adultes, ne peut, en toute bonne foi, prétendre écrire pour ses pairs et en fonction d'un cercle restreint d'initiés. Ce qui ne signifie pas pour autant que les auteurs jeunesse soient indifférents à la reconnaissance des pairs ${ }^{11}$. Cette reconnaissance se traduit avec le plus d'évidence par l'obtention d'un prix littéraire ${ }^{12}$ qui confirme, pour le lauréat, non seulement l'accumulation d'un "capital symbolique", dont souvent la valeur n'est reconnue qu'à l'intérieur du sous-champ, mais également un sentiment d'appartenance à une communauté restreinte qui partage des intérêts communs et spécifiques. Les propos d'Angèle Delaunois, lauréate du Prix du Gouverneur général du Canada, apparaissent à cet égard très significatifs ${ }^{13}$. Ajoutons que certains auteurs ou éditeurs occupent parfois des créneaux spécifiques ${ }^{1 / i}$ profilés de telle manière que ceux-

11. Une reconnaissance de la part des pairs à lintérieur du sous-champ est toutefois possible dans le mesure où les principaux prix littérairess (Gouverneur général, Christie, Cécile-Gagnon), hormis les prix de CommunicationJeunesse, sont attribués par d'autres acteurs du milieu.

12. Pour un historique des prix littéraires, lire Noëlle Sorin, "Historique des prix littéraires du Québec attribués à la littérature jeunesse", Canadian Children:s Literature/Littérature canadienne pour la jeunesse (CCI/LCI), Guelph (Ontario), $n^{125} 100 / 101$, hiver 2000/printemps 2001, p. 69-93.

13. Voir ces propos recueillis par Jean-Denis Côté, dans "Angèle Delaunois: lauréate du Prix du Gouverneur général du Canada", Canadian Cbildren's literature/Littérature canadienne pour la jeunesse (CCL/LCJ), Guclph (Ontario), n" 94, été 1999, p. 63: «Vous savez, le travail d'écrivain en est un de solitairc. Nous sommes seuls, enfermés dans notre bulle et tout dun coup, quand on gagne un prix comme celui-là, nous nous retrouvons sous les feux de la rampe. C'est à la fois très excitant et très dérangeant. Mais c'est un beau feeling puisque ça me donne beaucoup d'énergie pour continuer. Je me sens rassurée par le choix de mes pairs. Cela m'aide à máintenir une certaine confiance en moi. J'en ai besoin. Le contact avec les autres lauréats a ćté aussi très enrichissant. J'ai eu l'impression d'appartenir à une grande confrérie, à une belle continuité, de parler avec des gens qui vivaient de l'écriture comme je la vis, qui avaient la même passion et les mêmes exigences. Des gens que je n'aurais jamais probablement rencontrés autrement. C'était formidable.*

14. À titre d'exemple, mentionnons Michel Quintin (des Éditions Michel Quintin), surnommé "l'éditeur vert "en raison de l'importance qu'occupe, dans ses livres, le milieu naturel (les animaux, la forêt, les grands espaces), soit comme cadre à l'histoire, soit comme thème principal. De même, les Éditions 
ci comportent, par la force des choses, des contraintes pouvant conduire à une marginalisation et, par là, à une réduction de la part de marché. Mais, malgré tout, ne cherchent-ils pas eux aussi à rejoindre le plus large public possible?

J'ai posé la question sur laquelle s'ouvre cet article lors d'une enquête sociologique menée auprès d'une trentaine d'acteurs du sous-champ de la littérature jeunesse au Québec: auteurs ${ }^{15}$, éditeurs/directeurs de collection, professeurs/chercheurs à l'université, critiques littéraires, animateurs de lecture. Si le regroupement des acteurs dans le champ paraît ici assez clair, il importe de préciser qu'il fut difficile de procéder à une division des répondants, ceux-ci occupant, comme l'a déjà relevé Claire Le Brun ${ }^{16}$, différentes positions dans le sous-champ. La position d'éditeur est particulièrement symptomatique de ce phénomène, car tous sont à la fois éditeurs ou directeurs de collections et auteurs. Le fait d'occuper une double position lorsqu'on est éditeur ou directeur de collection dans le milieu n'apparaît donc pas comme relevant d'une situation exceptionnelle ou marginale, mais correspond à la norme, qui s'inscrit dans la pratique tel un invariant structurel. Le regroupement des acteurs a donc été établi en fonction de ce qui nous est apparu leur position principale dans le sous-champ.

Cette enquête menée auprès des acteurs du sous-champ de la littérature jeunesse a été amorcée à l'automne 1997. J'ai interviewé trente-six agents de ce sous-champ, soit dix-sept auteurs ${ }^{17}$, onze éditeurs et directeurs de collection ou ex-directeurs de collections ${ }^{18}$, trois professeurs/chercheurs (dont un est également auteur jeunesse), deux critiques littéraires (qui sont de surcroît auteur ou animateur de lecture), deux animateurs de lecture et un conseiller pédagogique.

du Soleil de minuit ont comme politique éditoriale de publier des livres qui représentent, soit dans le texte, soit dans l'illustration, des individus issus des communautés culturelles ou des Premières Nations.

15. Le générique masculin est utilisé pour alléger le texte et, surtout, pour favoriser l'anonymat des répondants.

16. Claire Le Brun, "Le roman pour la jeunesse au Québec. Sa place dans le champ littéraire ", Globe, Montréal, vol. 1, n², 1998, p. 57.

17. Voir Jean-Denis Côté, "Charlotte Gingras, lauréate du Prix du Gouverneur général du Canada 1999*, Canadian Children's Literature/Littérature canadienne pour la jeunesse (CCL/LCJ), Guelph (Ontario), $\mathrm{n}^{\circ} 98$, été 2000, p. 5567.

18. Une directrice de collection, également écrivaine, m'a autorisé à publier l'entrevue faite avec elle: voir note 13 
Le discours dominant que mon enquête permet de dégager est le suivant: si on écrit une cuvre avec comme souci essentiel de vendre des livres, si on bâtit une ouvre dans le but avoué d'obtenir un meilleur tirage, il y a contradiction. En revanche, si le but premier est d'écrire une bonne histoire, de parvenir à une œuvre réussie, et si, par ailleurs, on obtient un tirage intéressant, le fait que le livre devienne une source de revenus n'est pas perçu comme une contradiction. Bref, pour qu'aucune contradiction ne soit ressentie, il importe que l'argent ne soit pas ce qui motive, de prime abord, l'écrivain. Cette réponse d'un auteur qui a commencé à écrire pour les adultes avant de se consacrer surtout à l'écriture pour les jeunes, est, en ce sens, fort représentative du discours dominant:

Le profit n'est pas la motivation première. Si cela avait été le cas, je n'aurais jamais écrit. J'aurais tout de suite arrêté après le premier roman. J'aime écrire, mais pas au prix de la mendicité, bien sûr. Je ne m'attends pas, non plus, à devenir riche avec ce travail. Je crois que nous pouvons être heureux sans être riche. Il est évident que nous valorisons beaucoup l'argent. Quand nous en avons suffisamment pour manger et avoir un toit, cela suffit. La seule préoccupation que j'ai, c'est de me nourrir et me vêtir.

Plus on semble se détacher de préoccupations relatives à la vente de ses livres auprès du public auquel on s'adresse et plus on se rapproche d'une conception de l'oeuvre qui tend vers une conception de l'art pour l'art, faut-il remarquer ici, moins il semble y avoir de contradiction entre création et gain financier. Cependant, les réponses des acteurs varient selon leur position dans le champ (auteur ou éditeur) et la nature du lien qu'ils entretiennent avec le public qu'ils tentent de rejoindre (volonté d'intéresser le lecteur par une histoire de qualité ou celle de lui vendre un livre). Mais examinons maintenant ces réponses en fonction du découpage que l'on a annoncé précédemment et en commençant par les acteurs qui sont à l'origine de la création, c'est-à-dire les auteurs.

\section{Les auteurs}

À la question qu'on leur posait, presque tous les auteurs ont répondu "non", à une exception près. Le seul à avoir répondu d'emblée qu'il y avait "sûrement" une contradiction était aussi le 


\section{Tangence}

40

seul, au moment où l'on procédait aux entrevues, à poursuivre des études doctorales en littérature à l'université. Ce fait m'est apparu significatif, dans la mesure où sa réponse n'est vraisemblablement pas étrangère au fait de fréquenter le milieu universitaire, surtout à un niveau où on privilégie tout particulièrement la recherche et, par conséquent, des questions de cet ordre.

Cet auteur, à l'instar des autres, s'empresse toutefois de nuancer sa réponse en ramenant la question à des préoccupations d'ordre pratique :

On vit dans le monde que l'on voudrait bien changer. Mais on vit quand même dans un monde où tout ne nous est pas donné gratuitement, où il faut un minimum pour vivre. Je ne sais pas chasser et je n'ai pas envie de faire pousser des graines. Ça me prend un revenu. $\grave{A}$ mon sens, pourquoi ne serais-je pas rémunéré pour un travail, le travail de création, qui est ni plus ni moins noble qu'un travail manuel (si j'étais plombier) ou un travail d'économiste? [...] La noblesse et les grands idéaux très naifs voudraient que ça se fasse au-dessus de l'argent et du profit. Mais dire: "J'écris pour la postérité", ça ne paie pas le loyer.

Il indique aussi que, si le milieu littéraire comporte son lot de contradictions, il n'est pas différent des autres à cet égard: "Nous sommes pour l'égalité des peuples et contre l'exploitation. Pourtant, nous participons à l'exploitation du Tiers-Monde par le "Premier-Monde" par le simple fait d'y vivre".

Deux auteurs ont mentionné qu'ils auraient très certainement répondu "oui" à la question lorsqu'ils étaient plus jeunes ou étudiants à l'université. L'un d'entre eux n'adopte cependant plus le même point de vue sur la question, affirmant désormais que les gains financiers, loin d'être une source de contradiction pour le créateur, pouvaient l'aider dans son travail en lui procurant une liberté, une tranquillité d'esprit:

Plus jeune, [...] j'aurais dit qu'il faut faire de l'art pour l'art. J'aimerais beaucoup pouvoir vivre à partir de mes redevances de livres, car je souhaiterais consacrer davantage de temps à cette activité. J'aimerais pouvoir le faire autrement que le soir ou lorsque je suis en tournée en Abitibi et que je n'ai rien d'autre à faire. Je suis pigiste, ce qui, je crois, est pire que d'avoir un autre emploi. Autrement dit, je fais de la traduction, de la correction, des textes sur commande. Ça tourne toujours autour de l'écriture. Je réalise des tournées dans les écoles. Pour arriver vraiment à vivre et payer le minimum dont on a besoin, c'est 
essentiel. L'écriture est reportée dans les moments où je n'ai vraiment rien à faire. Lorsque je suis pigiste, j'ai même des contrats la fin de semaine! Je trouve donc difficile de concilier l'écriture avec une telle activité professionnelle. Lorsqu'il y a des bourses, parfait, mais nous n'en avons pas tout le temps.

Pour cet écrivain, la bourse (ou, éventuellement, les gains financiers) peuvent permettre de se consacrer entièrement à la création. En conséquence, elle donne la possibilité, indirectement mais de manière déterminante, de favoriser le travail du créateur en raison de l'indépendance qu'elle procure.

L'autre écrivain à avoir changé d'avis sur la question m'a confié que sa conception de l'artiste détaché des préoccupations matérielles avait éclaté lorsqu'il a pris conscience qu'un bon nombre d'artistes, parmi les plus grands, créaient soit par obligation, soit parce qu'ils étaient protégés par un mécène: "Balzac écrivait pour payer ses dettes. Jean-Sébastien Bach avait une commande à remplir tous les dimanches et devait écrire une partition pour la prochaine messe dominicale. Greco peignait, mais il était protégé par certaines autorités de son époque, dont l'Église". Pour cet auteur, la conception de l'artiste détaché des nécessités du quotidien est factice, mais il insiste pour dire que l'on peut écrire en raison d'une "certaine nécessité, en se respectant soi-même". En guise d'exemple, il évoque un refus de sa part d'écrire des livres qu'un éditeur lui avait proposés. Le projet était tentant, car le choix du sujet lui assurait pratiquement la vente du tirage initial: "L'éditeur anticipait qu'il y avait un public gagné d'avance pour ce genre de livre. Il a décliné l'offre, car elle ne correspondait pas à ses visées en matière d'écriture. En revanche, il a accepté d'écrire des romans policiers pour le même éditeur parce qu'il affectionne cette forme romanesque.

Un autre écrivain, lui aussi animateur de lecture, précise qu'il pourrait y avoir contradiction, mais insiste pour dire que les créateurs méritent d'être rémunérés pour le travail qu'ils accomplissent et ne le sont sans doute pas suffisamment. Il regrettait de ne pouvoir vivre de sa plume. Lors de l'entretien, cet écrivain en a profité pour attirer mon attention sur une autre situation encore plus paradoxale selon lui. Il a évoqué l'exemple d'un autre écrivain qui, à l'occasion d'un salon du livre, est "sorti de ses gonds" en constatant que, de tous les individus travaillant au kiosque de son éditeur, il était celui qui recevait la plus petite rémunération. Parmi les intervenants dans la chaîne du livre, l'auteur est celui 
qui "touche le moins d'argent", alors qu'il est à l'origine du livre et que, sans lui, le livre n'existerait pas.

L'écrivain qui a répondu avec le plus de conviction qu'il n'y avait pas de contradiction entre création littéraire et gain financier est aussi l'un de ceux qui, selon des sources informelles, recevrait les redevances les plus importantes du milieu. Son travail de créateur lui permet de vivre de sa plume, grâce à la publication de ses nombreux romans jeunesse (plus d'une quinzaine). L'écriture est son activité principale. Voit-il une contradiction entre son travail de création et le gain financier?

Absolument pas. C'est une idée du dix-neuvième siècle, qui veut qu'il faille absolument souffrir lorsqu'on écrit ${ }^{11}$. Il ne faut surtout pas avoir de succès et il ne faut surtout pas être payé pour notre succès! Cela fait l'affaire des éditeurs. Ils ne sont pas obligés de payer les auteurs. Cela fait aussi l'affaire du gouvernement, qui refuse d'investir. Au moins, ils ont mis des lois afin que les auteurs soient les premiers payés lorsqu'il y a faillite chez un éditeur. En somme, ça [que l'on puisse penser qu'il y a it contradiction] convient à bien des gens. Nous gagnons notre vie en écrivant. Pour être un bon écrivain, il faut travailler, écrire. Nous ne faisons pas cela la fin de semaine entre deux brassées de lavage. Certains le croient, pourtant. Je gagne ma vie avec cette activité. J'ai une maison à payer, de l'électricité, du chauffage. Il n'y a aucune contradiction, c'est évident. Bien sûr, si vous êtes professeur à l'université et que vous touchez 80000 \$ par année pour votre travail, vous écrivez alors évidemment pour votre plaisir. Même si vous ne vendez que 300 exemplaires, ça importe peu. Votre vie est assurée. Les choses sont différentes si vous êtes auteur et écrivez à temps plein. D'abord, vous ne mettez pas trois ans à le faire. Il faut que ça sorte. Cela change un peu la manière d'écrire. Je produis beaucoup plus que je ne le ferais si j'écrivais pour mon plaisir. Par contre, je ne suis pas certain que cela serait de meilleure qualité. Pour bien écrire, il faut écrire. Pour être un bon patineur, il faut patiner. Vous n'avez pas le choix. Si j'écrivais seulement une fois de temps en temps, le livre ne serait peut-être pas bon. Le livre est peut-être bon parce que j'écris tout le temps. On

19. Gustave Flaubert à George Sand (12 décembre 1872), cité par Pierre Bourdieu, Les règles de l'art, ouvr. cité, p. 121 : "Quand on ne s'adresse pas à la foule, il est juste que la foule ne vous paie pas. C'est de l'économie politique. Or, je maintiens qu'une aeuvre d'art digne de ce nom et faite avec conscience est inappréciable, n'a pas de valeur commerciale et ne peut se payer. Conclusion: si l'artiste n'a pas de rentes, il doit crever de faim!* 
croit toujours que nous ne devrions pas être payés pour écrire, que nous sommes un service public. Je ne suis pas un service public. Si vous voulez me donner un salaire, tant mieux, je vais le prendre. Je dois vivre.

Un autre écrivain interviewé vit et a toujours vécu grâce à l'écriture, pour les adultes mais surtout pour les jeunes, et depuis quelques décennies déjà. À l'instar de l'auteur précédent, il ne voit pas de contradiction.

En principe, ce qui est de qualité devrait bien se vendre. Je gagne ma vie avec mon écriture. Je suis l'un des rares à le faire. L'écriture, c'est ce que je sais faire et je crois que je le fais bien. [...] Pour moi, profit et qualité vont de pair. Lorsque je travaillais à contrat, il fallait que je livre la marchandise. Si ça n'avait pas été bon, ça n'aurait pas été accepté. Je ne suis pas du genre artiste qui vit dans sa tour d'ivoire et qui se contente de peu. Je crois que l'on peut gagner sa vie honnêtement, avec l'écriture, à condition d'avoir plusieurs débouchés.

Lorsqu'il parle "d'avoir plusieurs débouchés", il évoque l'écriture de manuels scolaires et de scénarios pour des émissions télévisées, car il ne lui apparaît pas vraiment possible d'obtenir des revenus suffisants en se limitant à l'écriture de romans jeunesse.

Lors de mon enquête, un écrivain (un seul) s'est situé en marge de tous les autres en se montrant, dans une certaine mesure, détaché du profit. Il tire ses revenus uniquement de ses droits d'auteur et des tournées d'animation qu'il fait dans les écoles. Il vit, selon Statistique Canada, en dessous du seuil de pauvreté fixé à $12000 \$$ par année pour une personne vivant seule ${ }^{20}$. Certaines années, ses revenus se limitent à $6000 \$$, ce qui l'oblige à puiser dans ses économies des années antérieures pour "boucler les fins de mois". Il ne possède ni voiture ni permis de conduire. Il n'a pas l'impression de vivre "de façon inconfortable" ou que son "niveau de vie [est] misérable", mais reconnaît que ce sont là des conditions de vie minimales. Pour lui, la véritable richesse se trouve dans le travail qu'il accomplit, c'est-à-dire la création et l'animation:

20. Statistique Canada a changé sa terminologie et a adopté la formulation aseuil de faible revenu. D'après les données disponibles les plus récentes, le seuil de faible revenu en 1998 pour une personne vivant seule au Canada dans une agglomération urbaine de plus de 500000 habitants était fixé à 14510 \$ par année alors que, pour une personne vivant seule en région rurale, il était fixé à $9514 \$$ par année. Source: Service des renseignements de Statistique Canada. 
En fin de compte, je n'ai pas l'impression d'être pauvre et de souffrir. Je me situe en dessous du niveau de la pauvreté, mais je vis très bien, je fais exactement ce que j'ai envie de faire. Ce que je fais, je le ferais même si je n'étais pas payé. Pour moi, c'est cela, la véritable richesse. Pourquoi veut-on être riche? pour ne plus avoir à gagner sa vie à faire des choses que nous n'aimons pas et pouvoir nous consacrer à $100 \%$ à nos loisirs. Eh bien, c'est littéralement ce que j'ai atteint en vivant en dessous du niveau de pauvreté! Je fais présentement ce que je ferais même si je n'avais pas d'obligation de gagner ma vie. J'aime écrire des livres, faire des bandes dessinées, des conférences. J'adore parler, comme présentement, du milieu littéraire. Et coincidence, des gens sont prêts à me payer pour faire ça. Pour moi, c'est du glaçage sur le gâteau. J'adore ça. Selon moi, je suis déjà riche.

À l'évidence, voilà un écrivain qui ne vit que pour son art. Bien qu'il souhaite sincèrement voir ses livres se vendre, il n'est pas prêt, dans l'unique but de répondre aux exigences d'un éditeur ou d'une demande préexistante du public, à écrire des romans, pas même un seul, qui ne correspondraient pas à ce qu'il a envie d'écrire:

Les livres insipides, pour lesquels je n'aurais pas à me battre, m'intéressent moins. Je n'ai pas envie d'écrire de telles choses pour des seules fins alimentaires. [...] Je n'ai pas envie de devenir quelqu'un qui se sent páuvre parce qu'il est obligé de faire ce travail afin de gagner sa vie. Je sentirais alors le fait de vivre en dessous du niveau de pauvreté et ça deviendrait lourd à supporter. Présentement, ce n'est pas le cas, puisque je fais ce que j'aime. J'ai du plaisir à écrire, à me consacrer à des causes auxquelles je crois.

Écrire des livres dans le but avoué d'obtenir un gain financier non seulement lui apparaît comme une contradiction en soi, mais viendrait marquer la fin de ce qui donne sens à son action, voire à son existence. Écrivain engagé et marginal, il choisit de défendre au quotidien des causes dont on retrouve naturellement l'écho dans ses livres. Cette attitude n'est pas sans provoquer quelques controverses, car le caractère audacieux des sujets abordés par l'écrivain choque parfois les intervenants du milieu scolaire. Certains sont même allés jusqu'à lui demander à la dernière minute de changer le sujet des animations qu'il devait faire dans leur école. Ils ont aussi refusé de distribuer ses livres aux élèves comme ils en avaient d'abord convenu, se livrant ainsi à une rupture de contrat et à une forme de censure. Ce sont les sujets et les 
pages couvertures de certains de ses livres qui auraient irrité des intervenants du milieu scolaire, indépendamment du fait que le coût des livres ait été entièrement défrayé par le ministère de la Culture et des Communications du Québec et que ceux-ci aient remporté des prix littéraires. Malgré ces difficultés, cet écrivain n'entend nullement changer sa vision des choses. En revanche, les revenus qu'il tire de ses droits d'auteur ne lui permettant pas de subvenir à ses besoins, il se voit dans l'obligation de devenir l'équivalent de son propre agent et doit, de ce fait, assurer la promotion de ses livres à la faveur des conférences qu'il prononce dans les écoles.

Le métier dauteur passe pour prestigieux, aux yeux des néophytes, alors que nous en arrachons à mort. Disons qu'il est plus facile de gagner sa vie comme auteur jeunesse que comme auteur adulte. Mais, même dans le premier cas, c'est loin d'être le Pérou. Et encore, rares sont ceux qui peuvent gagner entièrement leur vie grâce à l'écriture. Si je m'inscris parmi ces privilégiés, ce n'est pas parce que je gagne beaucoup [d'argent], mais bien parce que j'ai besoin de peu. Entendons-nous: il faut savoir gérer ses dépenses et ses revenus et également bien savoir se placer les pieds. Contrairement à d'autres écrivains qui se "consacrent entièrement à leur art", je suis l'un de ceux qui passent entièrement [leur] temps à se vendre. J'ai des dépliants annonçant mes conférences, je fais un publipostage tous les deux ans dans plusieurs bibliothèques publiques du Québec ou du Canada afin d'annoncer mes services. J'ai effectué des tournées un peu partout au Canada pour gagner ma vie. Il faut carrément que je me vende, que j'explique aux gens que je contacte: "Voici comment vous pouvez obtenir une subvention pour me faire venir." Je les prends par la main et je les guide. Les systèmes de subventions sont de plus en plus compliqués pour faire venir un écrivain. Vous ne pouvez plus inviter un écrivain pour une seule rencontre. Au Conseil des arts du Canada, vous devez commander au moins quatre rencontres du même auteur, ou encore quatre rencontres d'auteurs différents. Si vous faites une demande pour moins de quatre conférences, votre demande n'est pas considérée par le Conseil des arts.

D'une part, cet écrivain épouse les valeurs caractéristiques de l'artiste qui a choisi d'être "voué à son travail de manière totale et exclusive $^{21}$ ". D'autre part, comme le rayonnement de son œuvre ne lui assure pas des revenus et des ventes très élevés, il doit

21. Pierre Bourdieu, Les règles de l'art, ouvr. cité, p. 115. 
consacrer la majeure partie de son temps et de ses énergies à faire de la promotion, bien que lui-même en soit venu à considérer cela comme une "partie du travail "qu'il ne fait pas "à contrecour".

\section{Les éditeurs/directeurs de collection}

La position des éditeurs, bien que nuancée elle aussi, diffère de celle des auteurs. Les éditeurs, en raison de leur position dans le sous-champ, sont particulièrement sensibles à la vente des livres et à la demande du public, que ce soit les jeunes, leurs parents ou les personnes travaillant dans le milieu scolaire. Un éditeur admet de facto la contradiction :

C'est la grosse réussite de [notre maison d'édition] d'être parvenu à vivre avec cette contradiction. Ça provient de mes origines modestes: mon père était briqueteur. Il était d'un milieu modeste, où on aimait bien la lecture, mais moi, le soir, alors que je complétais mon bac, je lisais Le Joumal de Montréal. Je n'avais pas de bagage culturel. Pour moi, ça a toujours été : la culture oui, mais pour le plus grand nombre. Il y a toujours eu cette ambiguité-là. Il y a toujours une urgence. Rien n'est jamais gagné, il faut se battre pour obtenir ce que l'on veut. Pour moi, ça va être ça dans les prochaines années, cette combinaison: présenter un bon contenu et rejoindre un large public. Tu vois, des artistes comme Robert Lepage, Carbone 14, La La La Human Steps, le Cirque du Soleil, qui est un précurseur dans son domaine, font des choses apparemment difficiles, mais obtiennent de gros succès internationaux. Ce phénomène d'artistes qui ont du contenu et qui rejoignent aussi des gens s'implante, mais ce n'est pas le principe de "l'audimat " de la télé où, s'il n'y a pas 1800000 personnes, on te dit "Dehors " et où on est prêt à faire n'importe quel compromis pour rejoindre tout le monde. Je ne pense pas rejoindre tout le monde et je ne le veux pas. Mais je vais essayer de rejoindre chaque personne potentielle. Tu vas aller en Gaspésie, aux Îles de la Madeleine, chez Jean Coutu, chez Zellers, chez Wall-Mart, tu vas voir nos livres. Les gens les voient: ils sont accessibles aux gens de toutes les classes sociales. On peut avoir de bons lecteurs dans des milieux humbles comme on peut avoir de mauvais lecteurs dans des milieux aisés. C'est cela, ma philosophie. Je suis probablement plein de contradictions: j'ai réussi à écrire, malgré le fait que je sois le président d'une grosse entreprise. Tant que cet équilibre ne sera pas rompu, je serai très bien. Si je n'arrive pas à écrire, je vais devenir tellement frustré que ça ne marchera pas. Il faut aussi que je puisse écrire. 
Si les autres éditeurs sont plus réticents à parler de contradiction, en disant simplement qu'il faut bien "gagner sa croûte", certains vont jusqu'à remettre en question le fait qu'ils se situent dans le champ de l'art, qu'ils produisent des œuvres d'art, rattachant principalement ce concept aux auteurs non seulement de littérature destinée aux adultes, mais reconnus par l'institution, comme Anne Hébert. Ils considèrent davantage leurs propres livres comme des produits culturels. Une telle position n'étonne pas, car elle est typique de l'éditeur se situant d'emblée à l'intérieur du champ de grande production symbolique.

En revanche, un ex-directeur de collection, qui se consacre entièrement à l'écriture depuis quelques années, tend à adopter la position des auteurs et du discours dominant, rappelant que l'argent n'aurait pu être sa motivation première. Un autre directeur de collection qui, lui, a pratiquement cessé d'écrire de la fiction, indique de manière nuancée qu'il "n'y a pas de contradiction, mais pour cela, l'argent ne doit pas être le seul moteur". Un autre directeur de collection, conscient d'émettre une position d'éditeur, mentionne l'importance des profits pour l'éditeur puisque, sans eux, il n'y a pas de livre, donc pas de littérature et, si on suit la logique de la théorie de Bourdieu, pas même de créateur:

Il suffit de poser la question interdite [Qui a créé ce "créateur"?] pour apercevoir que l'artiste qui fait l'œuvre est lui-même fait, au sein du champ de production, par tout l'ensemble de ceux qui contribuent à le "découvrir "et à le consacrer en tant qu'artiste "connu " et reconnu - critiques, préfaciers, marchands, etc. Ainsi, par exemple, le commerçant d'art (marchand de tableaux, éditeur, etc.) est inséparablement celui qui exploite le travail de l'artiste en faisant commerce de ses produits et celui qui, en le mettant sur le marché des biens symboliques, par l'exposition, la publication ou la mise en scène, assure au produit de la fabrication artistique une consécration d'autant plus importante qu'il est lui-même plus consacré. Il contribue à faire la valeur de l'auteur qu'il défend par le seul fait de le porter à l'existence connue et reconnue, d'en assurer la publication (sous sa couverture, dans sa galerie ou son théâtre, etc.) en lui offrant en garantie tout le capital symbolique qu'il a accumulé, et de le faire ainsi entrer dans le cycle de la consécration qui l'introduit en des compagnies de plus en plus rares et recherchées $[\ldots]^{22}$.

22. Pierre Bourdieu, Les règles de l'ant, ouvr. cité, p. 238. 


\section{Les critiques}

Les critiques de la littérature jeunesse qui, par leur simple existence, contribuent à l'institutionnalisation de ce sous-champ en lui donnant des lettres de noblesse auprès du lectorat adulte, adoptent des positions qui se révèlent un peu différentes. Ce phénomène s'explique sans doute en partie par le fait que l'un d'entre eux est aussi auteur jeunesse. Ce dernier n'y voit pas de contradiction, mais nuance son propos en s'inscrivant tout à fait à l'intérieur du discours dominant qui lui fait adopter le point de vue des auteurs: "Lorsque j’ai commencé à écrire, ce n'était vraiment pas pour gagner de l'argent. Je ne me faisais aucune illusion là-dessus. Je me rends compte que ça paie: tant mieux. Ce n'était absolument pas mon but premier, qui était plutôt de trouver un éditeur et d'avoir un livre sur lequel mon nom apparaitrait".

Pour l'autre critique, qui enseigne également la littérature jeunesse à titre de chargé de cours, il y a contradiction,

mais cette contradiction existe dans la vie de tous les jours. Comment voulez-vous qu'elle ne se retrouve pas dans les livres? Les gens veulent être de jeunes cadres dynamiques et efficaces, [dans le but de gagner] de l'argent. Ils vont faire tout ce qu'il faut pour y arriver, tout en se contraignant aux moules, parce qu'il faut plaire pour réussir. Cependant, en littérature, il faut savoir qu'il y a cleux types de réussite. Il y a les livres qui gagnent des prix, ou ceux qui devraient en avoir gagné puisqu'ils en méritaient, et il y a ceux qui se vendent bien. Parfois, il peut arriver qu'une problématique soulevée par quelqu'un arrive à toucher tout le monde, à faire l'unanimité ${ }^{23}$. Mais, fondamentalement, il y a une contradiction, sauf qu'il y a une petite frontière entre le fait d'être l'un et l'autre à la fois. Nous pouvons avoir un succès de librairie et avoir écrit une grande œuvre littéraire. Vous pouvez avoir visé dans le mille avec tout ce qu il fallait pour que ce soit éclatant.

La dichotomie établie par ce critique ("Il y a les livres qui gagnent des prix [...] et ceux qui se vendent bien ") montre, une fois de plus, les voies divergentes entre le champ de production restreinte et le champ de grande production symbolique, bien

23. Un bel exemple de roman jeunesse qui à reçu à la fois une reconnaissance populaire (prix des Livromaniaques de Communication-Jeunesse) et institutionnel (prix du Conseil des arts du Canada) est le livre de Raymond Plante, Le dernier des raisins, Éditions Québec/Amérique, 1986, puis Boréal, 1991. 
qu'en littérature jeunesse, l'écart ne soit pas aussi prononcé qu'en littérature générale. En effet, certains auteurs (Dominique Demers, Raymond Plante, Denis Côté, Robert Soulières) remportent les prix les plus prestigieux tout en voyant leurs titres atteindre des tirages élevés. Ces deux voies de consécration viennent également rappeler le rôle du critique en littérature jeunesse: faire une critique "littéraire", rigoureuse, indépendante du succès commercial que revendiquent bruyamment certaines ceuvres jeunesse.

\section{Les professeurs/chercheurs}

Précisons d'emblée que la situation des professeurs/chercheurs interviewés diffère, leur rapport au sous-champ de la littérature jeunesse n'étant pas du même ordre. L'un des trois est professeur en psychoéducation et écrit pour les jeunes, de sorte que tout porterait à croire que sa connaissance ou son intérêt pour le sous-champ de la littérature jeunesse au Québec est très prononcé. Mais tel n'est pas le cas. À plusieurs reprises, je me suis retrouvé dans la position de la personne interviewée, le professeur multipliant les questions à mon intention. Cela peut d'abord surprendre, mais se comprend ensuite aisement quand on sait que ses livres comportent une dimension didactique essentielle. En fait, et l'auteur/professeur ne s'en cache pas, les histoires racontées servent de support à un enseignement auprès du public, que ce soit le jeune lecteur ou ses parents. Par conséquent, la présence de cet agent dans le milieu peut apparaître accidentelle. D'ailleurs, pour lui, le problème des liens qui se nouent entre création et gain financier ne se pose pas, puisqu'il considère que les revenus générés par ses droits d'auteur ne lui permettraient pas de vivre de sa plume:

Par exemple, je récolte $0,60 \$$ par livre qui se vend, $10 \$$ ou 11 \$. Le marché au Québec est extrêmement petit. Nous vendons 5000 exemplaires de chaque livre de la collection [...] par année. C'est peu. Je ne connais pas de gens qui vivent de ça. Je n'aspire pas à ça. Bien sûr, il est important de faire ce que je fais, j'aime ça. Je pourrais me comparer à un historien qui aime écrire des livres d'histoire. J'ai accès à une documentation, je fais mes recherches, j'écris une histoire. Je crois que quelqu'un qui écrit un roman historique doit procéder un peu de la même façon. C'est un peu le statut que je me donne: un spécialiste qui aime écrire dans son domaine. 
Les deux autres professeurs/chercheurs interviewés consacrent l'essentiel de leurs travaux de recherche à la littérature jeunesse et l'enseignent dans leurs universités respectives. Ils ont donc une bonne connaissance de la production littéraire, de l'histoire de cette littérature, des acteurs du milieu. Leur point de vue m'apparaissait particulièrement intéressant, compte tenu du recul, de la distance et de l'indépendance que leur confère leur statut de chercheurs universitaires. Ces deux professeurs/chercheurs ont des positions très proches et celles-ci témoignent d'une proximité avec le discours dominant. Pour l'un d'entre eux, "produire une œuvre d'art afin d'en retirer un profit immédiat implique une contradiction. Si vous produisez une collection d'ceuvres d'art pour avoir un profit à long terme, parce que ceux qui vont avoir pris connaissance de ces cuvres d'art au cours de cinq, six, sept ans, vont toujours vouloir y revenir afin de mieux connaitre l'art, non, il n'y a pas de contradiction.

Quant à l'autre professeur/chercheur, il apporte les nuances suivantes:

Ce n'est pas parce que nous faisons une ceuvre littéraire de qualité que nous n'allons pas gagner d'argent. Il ne me semble pas que ce soit lié. Ce qui se procluit, c'est que si nous produisons une ceuvre avant-gardiste ou différente, clle peut venir trop tôt dans la société, avant que les gens soient prêts à accueillir ce type d'oeuvre. À ce moment-là, ça tombe à plat. Elle ll'ouvrel peut même être décriée, critiquée. À première vue, il me semble qu'il n'y a aucune contradiction entre produire quelque chose de qualité et gagner de l'argent. Il y a toutes sortes de détails qui peuvent faire une différence, dont celui de la réception de l'oulvre. Par contre, je sais que nous pouvons faire beaucoup plus d'argent si nous flattons le public dans le sens du poil, si nous lui offrons des choses à son goût. Le goût du public n'est pas nécessairement le sommet de la qualité littéraire ou artistique. L'art commercial est généralement un art inférieur, dans la mesure où il manque d'originalité, ou alors il n'est pas suffisamment soigné, travaillé. Il n'y a pas d'incompatibilité entre produire une ouvre et obtenir un gain. Mais, dans les faits, nous avons plusicurs exemples où qualité et gains monétaires ne vont pas de pair, parce que, justement, nous voulons trop faire "commerce" et pas assez "art ". 


\section{Les animateurs de lecture}

Les positions des deux animateurs de lecture interviewés divergent quelque peu. Si les deux affirment de prime abord une absence de contradiction, l'un se situe tout à fait à l'intérieur du discours dominant en soulignant que l'argent ne doit pas être ce qui motive l'auteur dans son travail. L'autre évoque néanmoins la possibilité que la recherche d'un gain financier puisse nuire à l'acte créateur, l'artiste, dans une société capitaliste, n'ayant peutêtre "pas le choix de produire ce qui se vend. En ce sens, il y a peut-être une contradiction. L'artiste n'a plus le loisir de s'exprimer autant qu'il le voudrait". L'exigence de rentabilité auquel se soumet l'éditeur serait susceptible de contraindre le créateur en l'obligeant à créer en fonction de la demande du public, ce qui aurait pour effet de limiter sa liberté d'expression.

Une des caractéristiques du sous-champ de la littérature jeunesse (si on le compare à celui de la littérature générale) serait justement le rapport à l'argent. Le fait que les acteurs du milieu de la littérature jeunesse parlent assez ouvertement d'argent apparaît comme un indice de leur appartenance marquée au champ de grande production symbolique. Cet argent, liê aux profits que génère la vente des livres, se ferait plus rare ces dernières années. Cet auteur explique le phénomène par la fragmentation du marché (un très grand nombre de livres publiés par année, entre 250 et 300 , au regard des capacités du marché) et par une certaine sclérose du milieu de l'édition que favorisent les subventions dont profitent les éditeurs. Ces subventions empêcheraient un renouvellement de l'édition jeunesse en lui interdisant d'adhérer pleinement à la logique qui gouverne le champ de grande production symbolique:

Les éditeurs de Comic Books [aux États-Unis], au lieu de rechercher la recette qui va fonctionner pendant les cinquante prochaines années, acceptent que le milieu de l'édition en soit un en constante mutation. Chaque année, chaque mois, nous y retrouvons de nouveaux produits [dont de nombreux produits dérivés], de nouvelles idées. Les choses avancent si vite que c'en est une véritable folie. Je n'affirme pas que nous sommes en mesure de faire cela [au Québec]. Par contre, on peut voir un exemple dans cette façon de procéder. Prendre un exemple ne signifie pas nécessairement de faire la même chose. Etre engoncé et être en perpétuel changement sont deux extrêmes. Il y aurait peut-être un certain équilibre à atteindre. 


\section{Tangence}

Je parle davantage ici de nouveaux concepts, afin que les éditeurs conscrvent la place qu'ils occupent. La seule manière, c'est de bouger constamment, cest le fourmillement d'idées, car il y a une forte compétition. Au Québec, le fait que les éditeurs soient subventionnés contribue à cet engoncement. Nous en discutons un peu dans le milieu, mais rarement ouvertement. Je ne dis pas que nous devrions abanclonner les produits actuels. Je ne suis pas du genre radical à lancer des idées sans y avoir réfléchi. Il serait absurde et inconséquent de dire: "Il faut que ça [le programme de subventions] arrêten. Par contre, peut-être y a-t-il des formules à changer. Lobjectif à viser, c'est d'insuffler une énergie dans ce milieu. Les subventions rendent les éditeurs trop amorphes. Bien sûr, il n'y aurait pas d'édition québécoise sans subventions. Il est merveilleux que nous soyons parvenus à nous doter d'une telle stnucture. Sauf qu'à présent, cette manière de procéder est l'une des causes de cette sclérose quil faut oser remettre en question. Ce que je vise, c'est qu'il y ait une littérature jeunesse vivante, branchêe, qui évolue constamment. Il faut dénicher cles moyens d'atteindre ce but. Si remettre en question le mode de financement actuel est lun de ces moyens, faisons-le.

Si ce que propose cet auteur se concrétisait, surviendrait dès lors ni plus ni moins qu'une petite révolution ou, si l'on préfère, une révolution partielle, au sein du champ. Les révolutions à l'intérieur des champs ne peuvent d'ailleurs qu'être partielles, puisqu'une révolution totale viendrait anéantir le champ lui-même. Au reste, on remarque la prudence du propos ("Il serait absurde et inconséquent de clire: "Il faut que ça [le programme de subventions] arrête." Par contre, peut-être y a-t-il cles formules à changer"), prudence qui montre à quel point cet acteur est conscient de l'ampleur du bouleversement qu'il suggère. Transposons ici à lorganisation de la production littéraire ce que dit Bourdieu des révolutions dans le contenu même de la recherche scientifique - ou de la création littéraire:

[...] de fait, les rétolutions partielles dont les champs sont continument le lieu no mettent pas en question les fondements mêmes du jeu, son axiomatique fondamentale, le socle de croyances ultimes sur lesquelles repose tout le jeu. Au contraire, dans les champs de production de biens culturels, religion, littérature, art, la subversion hérétique se réclame du retour aux sources, à l'origine, à l'esprit, à la vérité du jeu, contre la banalisation et la dégradation dont il a fait l'objet ${ }^{2 i}$.

24. Pierre Bourdieu, "Quclques propriêtés des champs", Quevions de sociologie, Paris, Minuit, 1980, p. 116; c'est moi qui souligne. 
En somme, l'étude dont on vient de faire état confirme l'appartenance des acteurs du sous-champ de la littérature jeunesse au champ de grande production symbolique, bien qu'ils partagent certaines pratiques propres au champ de production restreinte. Plus la position d'un acteur dans le champ est redevable au capital symbolique accumulé, lié à la qualité de sa production artistique, moins cet acteur semble y voir une contradiction. puisque le gain financier lui apparaît, il est vrai, non négligeable, mais secondaire, et survient comme par surcroit. Inversement, lorsque la position d'un acteur dans le champ est d'abord ou surtout redevable au capital économique, et par conséquent à la réception du public, immédiat (les jeunes) ou indirect (les parents et les usagers du milieu scolaire), on voit dès lors saffirmer deux points de vue distincts: il y a soit conscience de la contradiction et, du même coup, de la nécessité de l'assumer, soit volonté de láa nier en faisant comme si elle n'existait pas.

Les acteurs du sous-champ de la littérature jeunesse au Québec, interviewés dans le cadre de cette enquête, ne se situent donc pas, à une exception près, dans le champ de production restreinte où la création artistique se conçoit comme purement désintéressée. Cette attitude s'explique en partie parce qu'ils ne peuvent pas destiner leurs ouvres à un public restreint composé de pairs. La nature même de leur activité (écrire pour les jeunes) ne peut faire autrement que de les rendre plus sensibles à la reconnaissance populaire (le public lecteur), alors que se confiner à la reconnaissance des pairs viendrait, en quelque sorte, trahir les motivations (présumées véritables) de leur écriture. En revanche, s'ils se situent, par leur volonté de rejoindre un large public et par leurs préoccupations financières avouées, à l'intérieur du champ de grande production symbolique, ceux qui sont les plus détachés des préoccupations relatives à la rentabilité ou au gain et pour lesquels la création demeure le premier motif de leur activité se rapprochent du champ de production restreinte, du moins de l'esprit qui le caractérise. 